\title{
Informatics Methodology Used in the Web-Based Portal of the NASCITA Cohort Study: Development and Implementation Study
}

Michele Zanetti, BEng; Antonio Clavenna, MD; Chiara Pandolfini, PhD; Claudia Pansieri, PharmD; Maria Grazia Calati, HS; Massimo Cartabia, StatSc; Daniela Miglio, HS; Maurizio Bonati, MD

Laboratory for Mother and Child Health, Department of Public Health, Istituto di Ricerche Farmacologiche Mario Negri IRCCS, Milan, Italy

Corresponding Author:

Chiara Pandolfini, PhD

Laboratory for Mother and Child Health

Department of Public Health

Istituto di Ricerche Farmacologiche Mario Negri IRCCS

Via Mario Negri 2

Milan, 20156

Italy

Phone: 390239014478

Email: chiara.pandolfini@marionegri.it

\section{Abstract}

Background: Many diseases occurring in adults can be pinned down to early childhood and birth cohorts are the optimal means to study this connection. Birth cohorts have contributed to the understanding of many diseases and their risk factors.

Objective: To improve the knowledge of the health status of Italian children early on and how it is affected by social and health determinants, we set up a longitudinal, prospective, national-level, population-based birth cohort, the NASCITA study (NAscere e creSCere in ITAlia). The main aim of this cohort is to evaluate physical, cognitive, and psychological development; health status; and health resource use in the first 6 years of life in newborns, and potential associated factors. A web-based system was set up with the aim to host the cohort; provide ongoing information to pediatricians and to families; and facilitate accurate data input, monitoring, and analysis. This article describes the informatics methodology used to set up and maintain the NASCITA cohort with its web-based platform, and provides a general description of the data on children aged over 7 months.

Methods: Family pediatricians were contacted for participation in the cohort and enrolled newborns from April 2019 to July 2020 at their first well-child visit. Information collected included basic data that are part of those routinely collected by the family pediatricians, but also parental data, such as medical history, characteristics and lifestyle, and indoor and outdoor environment. A specific web portal for the NASCITA cohort study was developed and an electronic case report form for data input was created and tested. Interactive data charts, including growth curves, are being made available to pediatricians with their patients' data. Newsletters covering the current biomedical literature on child cohorts are periodically being put up for pediatricians, and, for parents, evidence-based information on common illnesses and problems in children.

Results: The entire cohort population consists of 5166 children, with 139 participating pediatricians, distributed throughout Italy. The number of children enrolled per pediatrician ranged from 1 to 100 . The 5166 enrolled children represent $66.55 \%$ $(5166 / 7763)$ of the children born in all of 2018 covered by the same pediatricians participating in the cohort. The number of children aged over 7 months at the time of these analyses, and for whom the most complete data were available upon initial analyses, was 4386 (2226/4381 males [50.81\%] and 142/4370 twins [3.25\%]). The age of the mothers at birth of the 4386 children ranged from 16 to 54 years. Most newborns' mothers (3758/4367, 86.05\%) were born in Italy, followed by mothers born in Romania (101/4367, 2.31\%), Albania (75/4367, 1.72\%), and Morocco (60/4367, 1.37\%). Concerning the newborns, 138/4386 $(3.15 \%)$ were born with malformations and 352/4386 (8.03\%) had a disease, most commonly neonatal respiratory distress syndrome $(n=52)$, neonatal jaundice $(n=46)$, and neonatal hypoglycemia $(n=45)$.

Conclusions: The NASCITA cohort is well underway and the population size will permit significant conclusions to be drawn. The key role of pediatricians in obtaining clinical data directly, along with the national-level representativity, will make the findings even more solid. In addition to promoting accurate data input, the multiple functions of the web portal, with its interactive platform, help maintain a solid relationship with the pediatricians and keep parents informed and interested in participating.

Trial Registration: ClinicalTrials.gov NCT03894566; https://clinicaltrials.gov/ct2/show/NCT03894566 
(J Med Internet Res 2021;23(3):e23087) doi: 10.2196/23087

\section{KEYWORDS}

internet; computer systems; cohort studies; pediatricians; infant; newborn

\section{Introduction}

It is well known that many diseases occurring in adults can be traced back to early childhood [1,2]. In fact, nearly all domains of later health experience, including cardiovascular and respiratory disease, cognitive decline, and psychological health, have been associated with early life exposures [3]. Many different factors in childhood play a role in future health inequalities between individuals, from socioeconomic status to parental care, to lifestyle factors, but the way they are related is uncertain.

Birth cohort studies are studies that follow a group of newborns for an extended period in order to assess possible associations between exposures in early life and later health. Northern Europe has a long-lasting tradition in birth cohorts [4,5], starting from as far back as 1921 [6]. Findings from these studies have led to important knowledge in different fields, contributing to the understanding of multiple diseases and their risk factors $[7,8]$. These studies have also set the basis of our positive daily health behaviors. The Avon Longitudinal Study of Parents and Children (ALSPAC), for example, showed that eating oily fish during pregnancy was associated with better eye and cognitive development in children [9].

Numerous large- and small-scale birth cohorts have been set up, also in the past decade, not only in Europe but all around the world [10]. Characteristics vary greatly from one cohort to another in terms of design, objectives, size, and duration of follow-up.

Since 2003, several cohorts have also been carried out in Italy. Most of them have general aims, with data collection limited in time or to specific geographical contexts [11-19]. Italy is a special country with a public, universal health care system that should be equally accessible to all, but considerable health inequalities exist $[20,21]$. Up to now, no national-level birth cohort has been set up that included a large sample of the pediatric population independent of socioeconomic status or other types of limitation, such as gestational age. The Piccolipiù cohort [17], for example, recruited newborns from northern and central Italy; the NINFEA cohort [16] population was limited to women who had enough knowledge of the internet to complete online questionnaires; and the ICON cohort [19] selected preterm newborns and enrolled additional newborns of later gestational age.

In order to improve the knowledge of the health status of Italian children early on and how it is affected by social and health determinants, we set up a longitudinal, prospective, national-level, population-based birth cohort, the NASCITA study (NAscere e creSCere in ITAlia) [22]. Like many other cohorts, it addresses multiple research questions [16,17]. NASCITA is unique, however, in terms of characteristics, methodology, and population size. The findings will add important evidence, in terms of epidemiological data, for the development of specific prevention measures and interventions to improve the health status of children.

The main aim of the NASCITA cohort is to evaluate physical, cognitive, and psychological development, and health status and health resource use during the first 6 years of life in a group of newborns, and to evaluate potential associated factors.

The peculiarity of NASCITA is that data collection is designated to the general pediatricians, fitting itself into the Italian public health care system, as data reported in NASCITA are part of those routinely collected by the family pediatricians at the well-child visits. Furthermore, the data are equally distributed throughout the Italian territory.

A website and web-based system [23] were set up in order to host the cohort, provide ongoing information to pediatricians and to families, and facilitate data input on the part of the pediatricians. The system was also designed to optimize data accuracy, minimize missing data, and permit data monitoring, analysis, and reporting throughout the duration of the cohort.

This article describes the informatics methodology used to set up and maintain the NASCITA cohort with its web-based platform, and provides a general description of the participant characteristics.

\section{Methods}

\section{Cohort Organization}

NASCITA is embedded in Italian pediatric primary care practice. Data collection for the NASCITA cohort occurs for the most part during the 7 well-child visits planned for each child. The majority of the participating pediatricians are part of the national Pediatric Cultural Association (ACP), an association with about 2000 members consisting mainly of family pediatricians and with which the coordinating center has collaborated over the years. Participation was proposed to the ACP and forms the basis of pediatrician participation in the study. Pediatrician participation was voluntary and for free. Collaboration was also expanded through contact with other pediatric scientific societies and associations. Meetings were held during 2018 to present the study to a group of pediatricians acting as local representatives. Each representative then asked other pediatricians working in their area (at the local health unit or regional levels) to participate. Pediatrician enrollment was monitored and discussed with the local representatives. A scientific committee was set up to supervise the project, and includes professionals and lay people from different fields of expertise.

At the start of the study, there were 7960 cities/towns in Italy. These were classified into 21 geographic clusters (Figure 1), identified based on geographic and administrative criteria used by the Italian National Statistics Institute (ISTAT) [24]. More specifically, these take into consideration geographic area (north, center, south), setting (urban, rural), and land characteristics 
(plain, mountain, sea). Four cities were also selected (Milan, Rome, Bari, and Palermo), covering the different geographic areas and the islands.

Enrollment of newborns began in April 2019 and ended in July 2020. Recruitment of the newborns (and their parents) took place during the first routine well-child visit scheduled for all newborns in Italy within their first 45 days of life. All newborns assigned to the participating pediatricians were enrolled if parental consent was given. Pediatricians chose when to begin enrolling their newborns and continued to enroll for (at least) a 1-year period. Follow-up of the children will continue until at least the age of 6 years.

A minimum recruitment of 5000 newborns was calculated in order to have enough power to study common childhood exposures and outcomes.

In this article we present the characteristics of the children aged over 7 months at the time of analyses in order to provide more complete data, as pediatricians would have had time to fill in most missing data for these participants.

Figure 1. The 21 geographic clusters, identified based on ISTAT geographic and administrative criteria. ISTAT: Italian National Statistics Institute.

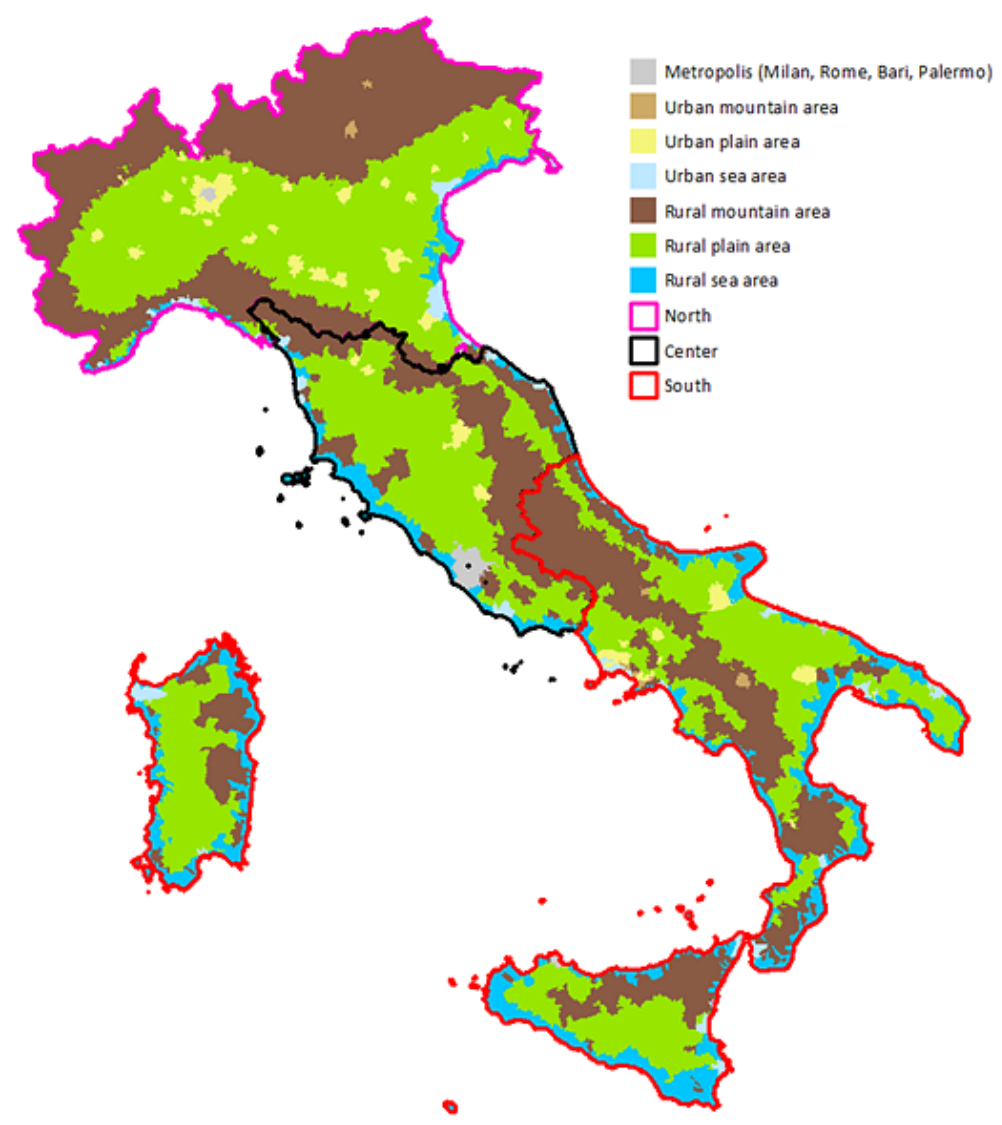

\section{Ethics}

Parents were given oral and written information about the study and a consent form to sign if interested in participating. Pediatricians signed a consent form before participation as well. Withdrawal from the study was guaranteed at any time to both pediatricians and parents.

\section{Data Collection}

Italian health care is provided free or at a nominal charge through a network of 148 local health units. The local health units assign children to a family pediatrician until they are 6 years old, after which the children can be registered with a general practitioner or remain with that pediatrician until they are 14 years old. In Italy there are about 7500 family pediatricians, for an average of 450,000 births/year [25], so about 60 newborns/year are assigned to each pediatrician. All children are scheduled 7 well-child visits at the pediatrician's office during their first 6 years of life to ensure necessary preventive care and monitor a child's growth and development.

Basic data are being collected and consist of data that are part of those routinely collected by the family pediatricians at the well-child visits. Some data will also be collected during each extra contact with the enrolled children. Data collection also involves parental data, such as medical history, characteristics and lifestyle, indoor and outdoor environment, and circumstances during pregnancy and around birth. Follow-up data on children will cover different fields, including physical and mental/cognitive development, nutrition and allergies, environmental exposures, and preventable infectious diseases. See Table 1 for a description of the main parts of the questionnaire. Questions were added to allow the project, in a second phase, to address specific areas such as nutrition, environment, and nurturing care. 
Table 1. Main sections of the online questionnaires and description of the general data collected.

\begin{tabular}{ll}
\hline Section & Description \\
\hline Personal data & Name, place of birth, family data such as number of family members, sibling health, parental place of birth, allergies \\
Medical history & $\begin{array}{l}\text { Mother's pregnancy data (including medicines, smoking and alcohol consumption, and reading out loud and lis- } \\
\text { tening to music), birth data (eg, newborn height, weight), perinatal medical history (eg, malformations, diseases, } \\
\text { transfer to an intensive care unit), breastfeeding status at discharge }\end{array}$
\end{tabular}

Visits $1-7$

Medicines taken, anthropometric measures; breastfeeding status/weaning/nutrition; sleep data; age-appropriate physical examination; vitamin D + K prophylaxis; psychomotor, neurologic, and cognitive development; general health; paternal depression; language development; family habits (eg, smoking, reading out loud, listening to music, nursery school, indoor and open-air activities); home proximity to traffic or to areas of intensive farming; screen time

Extra visits

Type of contact (office, phone, home visit), diagnosis, medicines/specialist visits/examinations prescribed

Vaccination compliance

Vaccines received and adverse reactions

Exiting the cohort

Reason for exit (transfer, pulled out of study, death)

\section{Statistical Analysis}

The analyses of the cohort data will evaluate specific research questions related to the overall aims of the study, such as the relationship between child development and the domains that affect nurturing care during the preschool period including health, nutrition, and caregiving routine; the association between the well-being of children and parental adherence to the recommendations for better child care and development; and the differences between geographical settings in educational and socialization opportunities available and in the care provided by the family pediatricians.

Data are presented as frequencies, percentages, and mean (SD) or median values. Percentages are based on denominators for which missing values have been excluded. All data management and analyses have been performed using SAS version 9.4 (SAS Institute) and ArcMap version 10.5 (ESRI). More detailed analyses will be performed, as specified in the protocol [22], and reported in future articles.

\section{Web Portal}

A specific web portal for the NASCITA cohort study was developed [23] with reserved sections for the coordinating center, registered users, and participating pediatricians. The web portal serves to assist pediatricians with data collection and to provide findings and other information during the study period to parents and pediatricians, also with the use of graphics for the analyses and data collected, based on a successful approach already reported by the coordinating center [26]. Selected sections of the portal have been translated into English. See Figure 2 for the functions of the web portal and its architecture.

Newsletters focused on child cohorts are periodically provided in the pediatrician's general area and contain bibliographic information of the current biomedical literature. In the private area, each participating pediatrician can access information including cohort documents; frequently asked questions; the study protocol; and pdf versions of the case report forms (CRFs); as well as patient data for input/modification; interactive data charts of his/her patients or of those of the entire cohort, including growth curves (Figure 3); and data concerning subsections of the cohort addressing areas such as nutrition and environment in which he/she participates. The pediatrician's section on the web portal, together with individual telephone calls with the pediatricians and online and in-person meetings on the study's progress and possible problems, serves to keep pediatricians engaged in the study.

The information for the parents section contains a growing series of cards, created in collaboration between health professionals and parents, that provide evidence-based information on the more common illnesses and problems in young children as well as answers to common questions that parents have on child care. This section also contains links to useful emergency telephone numbers and information pages. 
Figure 2. Functions of the platform and its architecture.

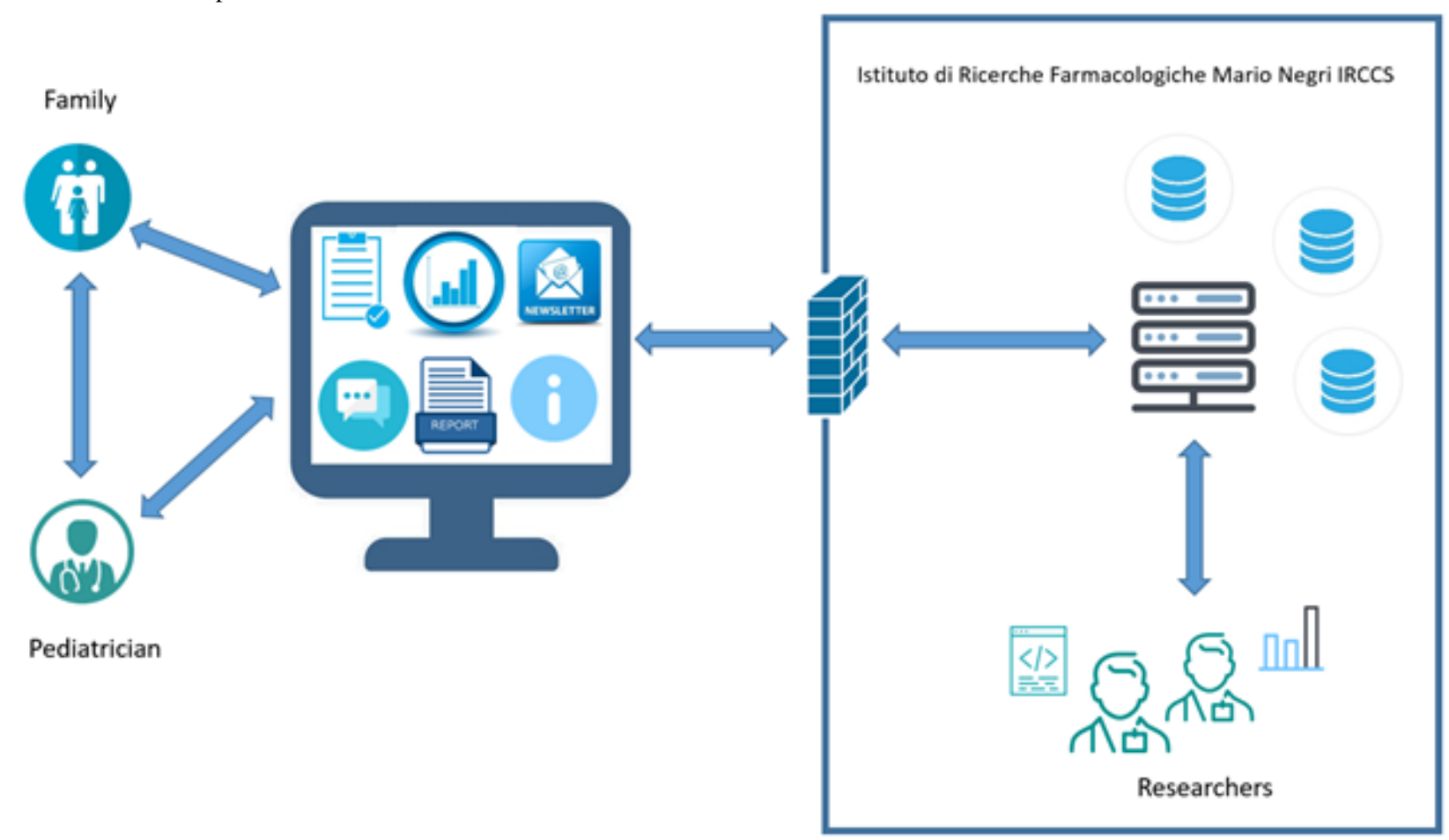

Figure 3. Individual child's data plotted on growth curve, as seen by the pediatrician.

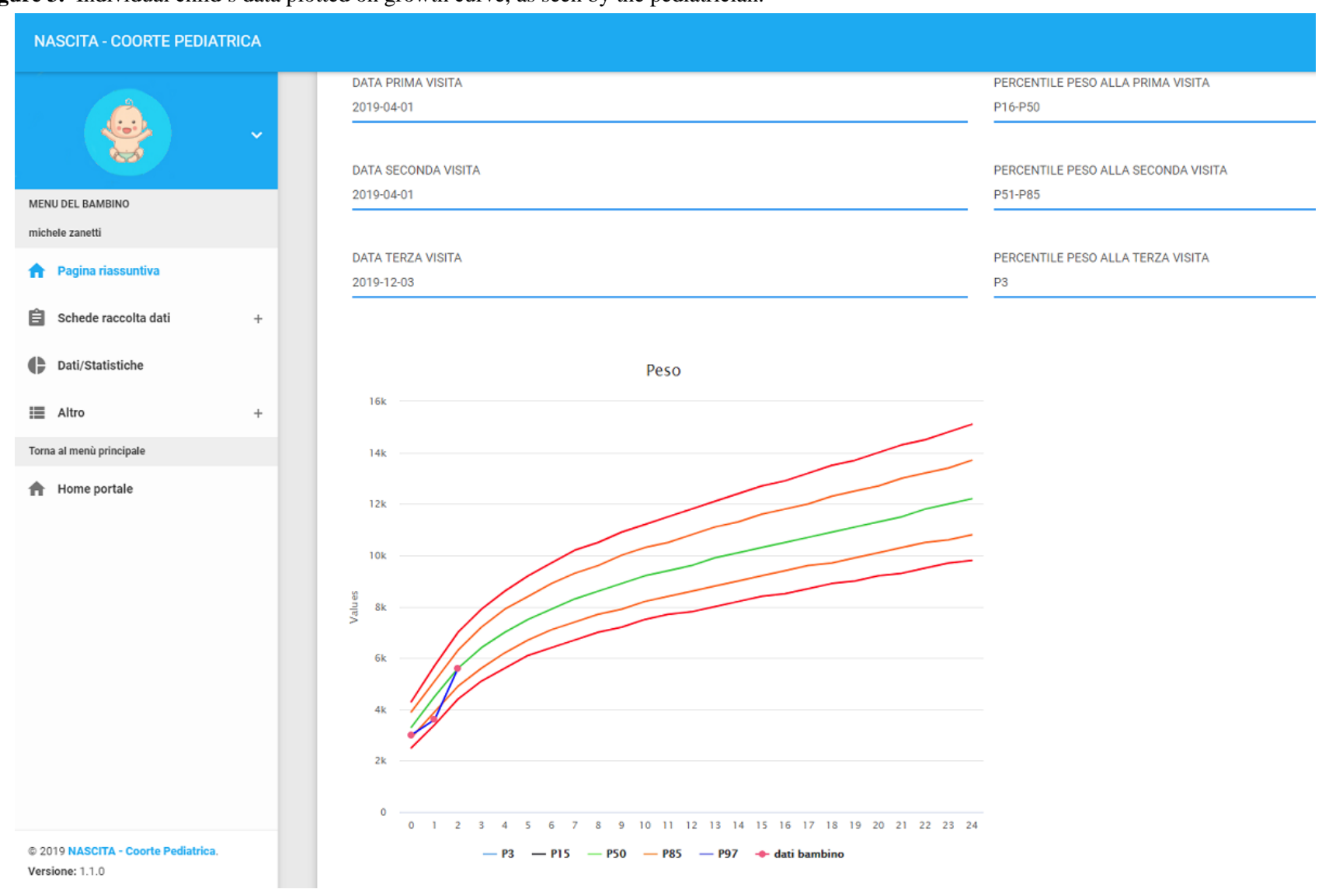

\section{Electronic Case Report Form}

The CRF was created and tested together with a group of pediatricians. An electronic CRF (eCRF) was then set up and tested, again with the help of the pediatricians, before enrollment began. More specifically, the pediatricians were asked to register themselves and access the portal starting from January 2019 to test it. The eCRF (Figure 4) was set up in such a way as to facilitate the pediatricians' input of data for the study and to provide fast and efficient support for any problems or doubts about data input. A "chat" section was consequently included through which pediatricians can ask for support. The eCRF 
includes consistency and range checks to prevent internal inconsistencies (eg, value ranges, fields with limited values, and time ranges). Data are, in any case, monitored continuously and irregularities resolved through email, chat, or phone contact with the pediatricians.

The eCRF has been structured in a way that will permit data collection to be expanded to cover the additional areas (eg, nutrition) more thoroughly in a second phase; the different data collection sections are, in fact, based on an XML definition that can easily be implemented and modified [27].

Figure 4. eCRF screenshots: input for second visit.
The development engine of the eCRF has been made available on Gitlab [28].

A test was performed with a group of pediatricians (including those with less experience in using the computer) to assess the additional amount of time it would take each pediatrician to enter data for a patient throughout the duration of the study. Entering data for the first follow-up visit took about 15 minutes. Multiplying this by the average number of newborns per pediatricians and, considering that after 3 months of the start of the study the subsequent regular check-ups would begin, an average of 3 hours a month in the first year was calculated, after which the amount of time necessary would decrease.

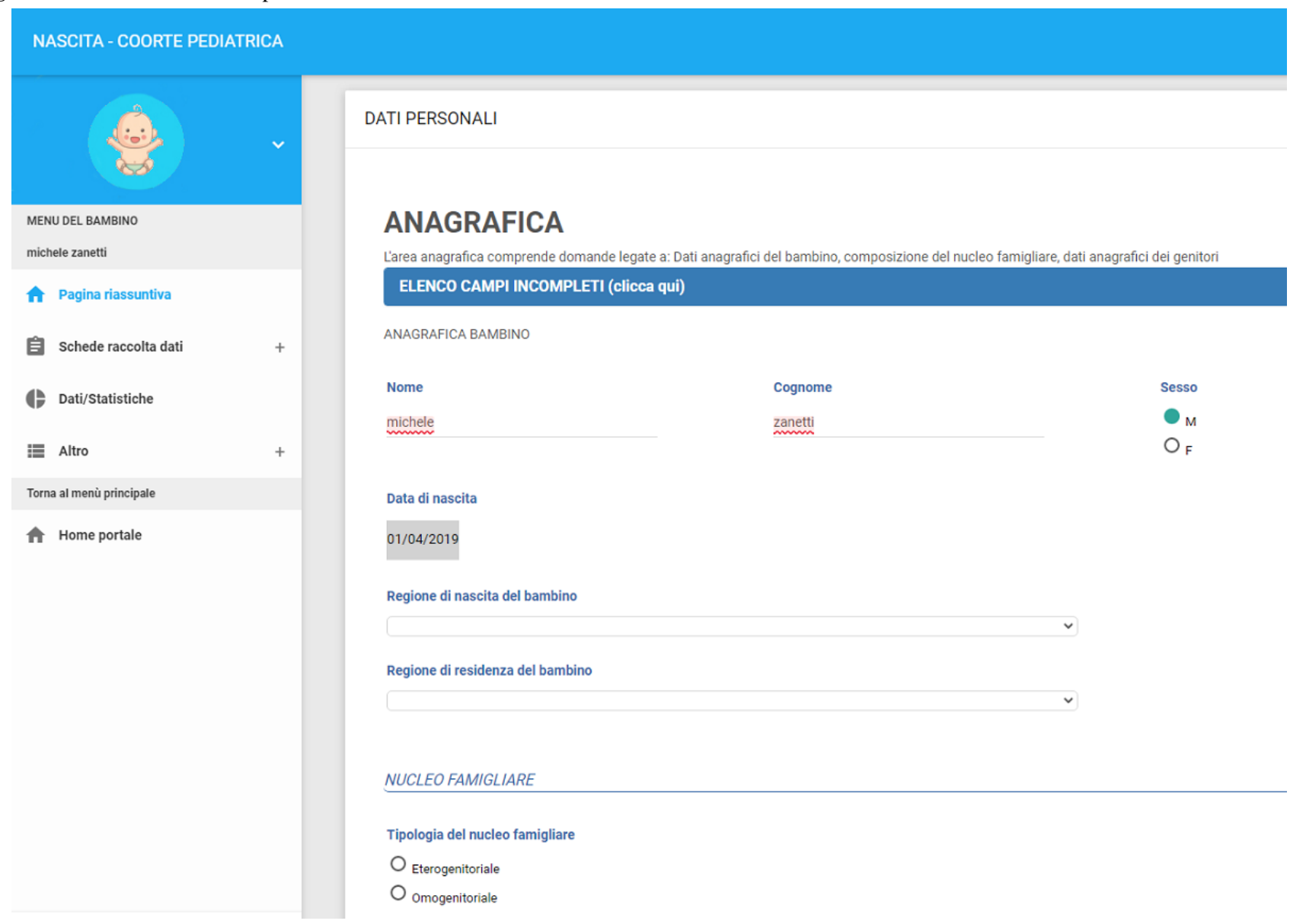

\section{Data Quality Control}

A dashboard is dedicated to checking the completeness, or lack, of the visits and displays a table listing the children for whom data have been included by that pediatrician. Each column in the table represents a specific visit and shows a series of colored bells (green, yellow, or red) that indicate the completeness status of the visit (Figure 5). When a pediatrician opens the data on a specific visit, the system displays a list of the variables with missing information in order to facilitate data completion.

Frequent reports will automatically be created to monitor recruitment of pediatricians and children and the data inputted. Individual and group reports will also be created for the pediatricians and for the study's scientific committee. 
Figure 5. eCRF dashboard for checking completeness of data on the visits for individual patients.

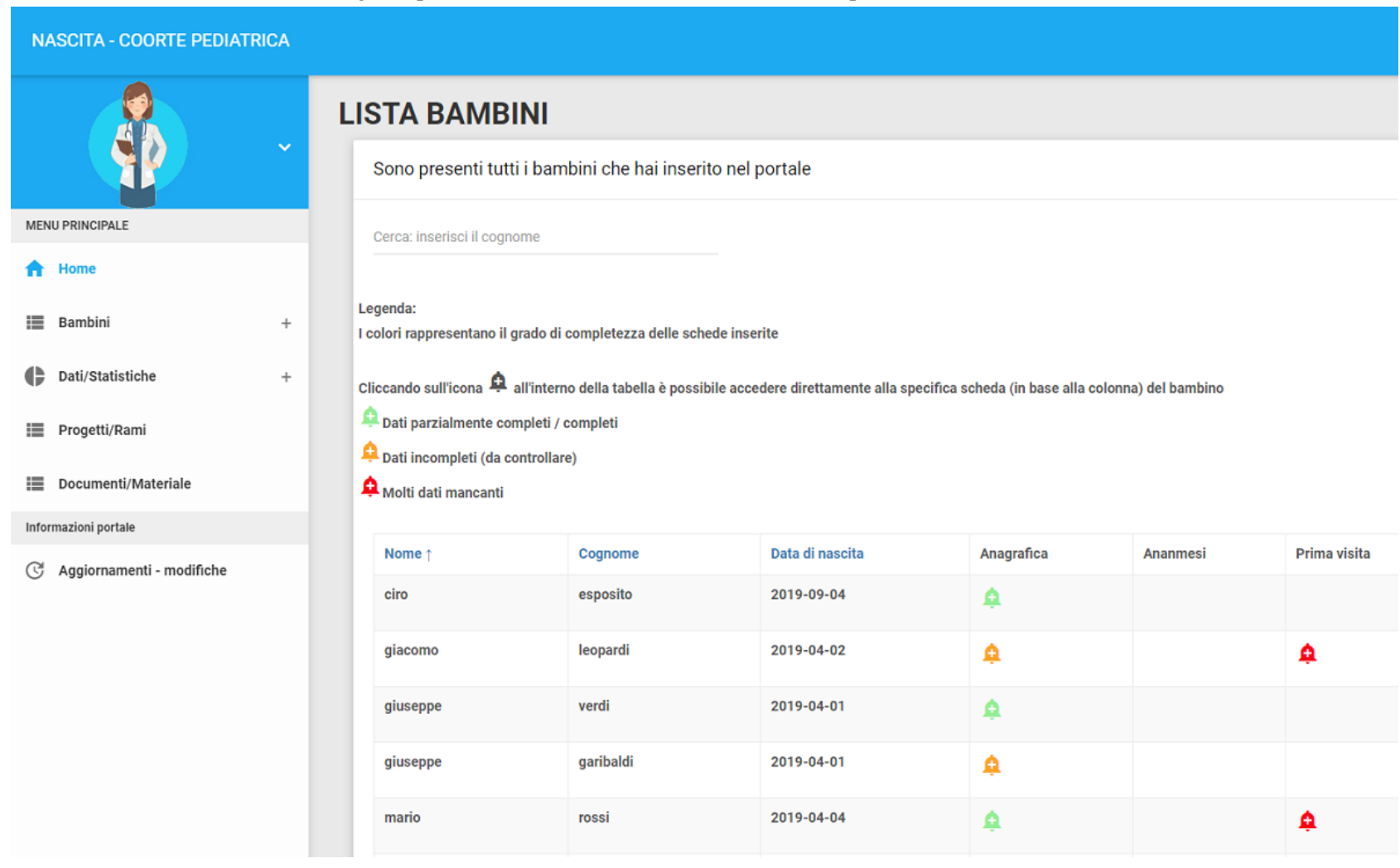

\section{Data Security}

The private area is accessible only through authentication by pediatricians, who have been previously approved and enabled. A specific role is assigned to them, for example, compiler. Passwords must have at least eight characters and contain special and uppercase characters. Each pediatrician with a compiler role can insert their patients' data. Sensitive data, such as name and last name, are encoded and visible only to the compiler. Once the data are saved, they are transmitted via secure HTTPS protocol, and are stored in databases that can be accessed only by authorized project staff (IT, statisticians). Back-ups are kept for security and disaster recovery.

\section{Results}

Enrollment in the NASCITA cohort began on April 1, 2019, and ended on July 31, 2020. The number of participating pediatricians is 139 and the total number of children enrolled is 5166. The pediatricians are distributed throughout Italy, with 68 in the north, 29 in the center, and 42 in the south. The 5166 children enrolled represent $66.55 \%$ (5166/7763) of the children born in all of 2018 covered by these same pediatricians.

The total number of children aged over 7 months at the time of these analyses was 4386 . Of these children, excluding those with missing data, 2226/4381 (50.81\%) were male and 142/4370 $(3.25 \%)$ were twins. The children were distributed throughout Italy, with 2025/4386 (46.17\%) in the north, 882/4386 (20.11\%) in the center, and $1479 / 4386(33.72 \%)$ in the south. Table 2 reports the distribution of the number of these 4386 children enrolled and the percentage of children born, by cluster and geographic area, based on ISTAT data [24], and shows that there are minimal differences. The number of children enrolled per pediatrician ranged from 1 to 100 (mean 32 [SD 18.5]; median 30).

The age of the mothers at birth of the 4386 children ranged from 16 to 54 (mean 33 [SD 5.4] years; median 33 years, excluding 133 missing values), while the age of the fathers ranged from 17 to 69 (mean 36 [SD 6.3]; median 36, excluding 154 missing values). Most of the newborn's mothers (3758/4367, 86.05\%) were born in Italy; the 3 next most common countries were Romania (101/4367, 2.31\%), Albania (75/4367, 1.72\%), and Morocco $(60 / 4367,1.37 \%)$. For two-thirds of children (2892/4320,66.94\%), the mothers were married or living in civil union; for $1233 / 4320$ children $(28.54 \%)$, the mother was living with the father; and for $167 / 4320(3.87 \%)$, mothers were single. Most of the children's mothers had a university $(1813 / 4320,41.96 \%)$ or high-school degree (1800/4320, $41.66 \%)$, followed by a middle-school diploma (675/4320, $15.63 \%)$ and an elementary (30/4320, 0.69\%) level of education. Family size (including the enrolled child) was grouped into 2, 3,4 , or $>4$ people, with half $(2189 / 4311,50.78 \%)$ of the families being made up of 3 people, followed by $1567 / 4311$ (36.35\%) made up of 4 people. Two-member families represented $1.43 \%$ $(62 / 4311)$ of the total. Concerning the pregnancies, 3741/4363 $(85.74 \%)$ were physiologic pregnancies, while in the remaining pregnancies gestational diabetes (203/622 mothers, 32.64\%), gestational hypertension $(90 / 622,14.47 \%)$, and preeclampsia $(41 / 622,6.59 \%)$ were the most common diseases. Concerning the newborns, $139 / 4340(3.20 \%)$ were born with malformations and $352 / 4335(8.12 \%)$ had a disease, the 3 most common of which were neonatal respiratory distress syndrome $(n=52)$, neonatal jaundice $(n=46)$, and neonatal hypoglycemia $(n=45)$. 
Table 2. Distribution of the number of children and the number of children born in each cluster and geographic area.

\begin{tabular}{|c|c|c|c|c|}
\hline Distribution/Location & $\begin{array}{l}\text { Children enrolled, } \\
\mathrm{n}(\mathrm{N}=4386)\end{array}$ & Population enrolled, n (\%) & Births per year in Italy ${ }^{\mathrm{a}}, \mathrm{n}(\%)$ & $\begin{array}{l}\text { Difference between the third and } \\
\text { fourth columns, } \%\end{array}$ \\
\hline Metropolis: Milan & 184 & $184 / 4386(4.20)$ & $11,267 / 467,640(2.41)$ & 1.79 \\
\hline Metropolis: Rome & 428 & $428 / 4386(9.76)$ & $21,497 / 467,640(4.60)$ & 5.16 \\
\hline Metropolis: Bari & 70 & $70 / 4386(1.60)$ & $2214 / 467,640(0.47)$ & 1.13 \\
\hline Metropolis: Palermo & 62 & $62 / 4386(1.41)$ & $5578 / 467,640(1.19)$ & 0.22 \\
\hline North: urban mountain & $-b$ & - & $2243 / 467,640(0.48)$ & -0.48 \\
\hline North: urban plain & 304 & $304 / 4386(6.93)$ & $45,595 / 467,640(9.75)$ & -2.82 \\
\hline North: urban sea & 93 & $93 / 4386(2.12)$ & $10,258 / 467,640(2.19)$ & 0.07 \\
\hline North: rural mountain & 228 & $228 / 4386(5.20)$ & $25,274 / 467,640(5.40)$ & -0.20 \\
\hline North: rural plain & 1151 & $1151 / 4386(26.24)$ & $112,824 / 467,640(24.13)$ & 2.11 \\
\hline North: rural sea & 60 & $60 / 4386(1.37)$ & $5882 / 467,640(1.26)$ & 0.11 \\
\hline Center: urban plain & 61 & $61 / 4386(1.39)$ & $6330 / 467,640(1.35)$ & -0.04 \\
\hline Center: urban sea & 1 & $1 / 4386(0.02)$ & $5339 / 467,640(1.14)$ & -1.12 \\
\hline Center: rural mountain & 90 & $90 / 4386(2.05)$ & $13,887 / 467,640(2.97)$ & -0.92 \\
\hline Center: rural plain & 233 & $233 / 4386(5.31)$ & $31,997 / 467,640(6.84)$ & -1.53 \\
\hline Center: rural sea & 68 & $68 / 4386(1.55)$ & $11,118 / 467,640(2.38)$ & -0.83 \\
\hline South: urban mountain & 8 & $8 / 4386(0.18)$ & $4010 / 467,640(0.86)$ & -0.68 \\
\hline South: urban plain & 136 & $136 / 4386(3.10)$ & $15,826 / 467,640(3.38)$ & -0.28 \\
\hline South: urban sea & 421 & $421 / 4386(9.60)$ & $27,281 / 467,640(5.83)$ & 3.77 \\
\hline South: rural mountain & 98 & $98 / 4386(2.23)$ & $21,932 / 467,640(4.69)$ & -2.46 \\
\hline South: rural plain & 421 & $421 / 4386(9.60)$ & $47,026 / 467,640(10.06)$ & -0.46 \\
\hline South: rural sea & 255 & $255 / 4386(5.81)$ & $40,262 / 467,640(8.61)$ & -2.80 \\
\hline Missing & 14 & - & - & - \\
\hline
\end{tabular}

${ }^{a}$ Based on data on the newborn population residing in the cluster on January 1, 2017. ISTAT demographic statistics data [24] referring to December 31, 2016, were used for clusters with missing data.

b_: not available.

\section{Discussion}

\section{Considerations}

The NASCITA cohort is based on community-level pediatric practice, involving the family pediatricians directly, as very few European cohorts do [29]. With their clinical practice, pediatricians are most in contact with patients and can promote study and action. Their involvement in child cohorts permits the collection of prospective, community-level data and allows them to contribute to optimizing both the quality of the data collected and its re-investment back into the community as health promotion interventions. In fact, pediatricians play a key role both in educating families and in implementing curative and disease prevention interventions through their routine clinical practice. They are in the optimal position to influence public health in general because adult health also depends on habits embraced when young, and pediatricians can undoubtedly influence children and their parents to adopt healthy lifestyles. In order to give something back to the pediatricians participating in the cohort, we have attempted to provide the pediatricians with useful information and interesting data, such as the interactive data charts of their patients. The system set up through which pediatricians can easily and quickly contact the cohort team for any questions or problems, and the periodic meetings organized to update pediatricians on the cohort's status and to discuss any current issues or suggestions are additional ways to show our appreciation for all their continuing efforts. During the latest meeting we had with the participating pediatricians, online in November 2020, we described the enrolled population as it was just after enrollment closure and the next steps. On this occasion several pediatricians provided additional suggestions for improving input, resulting in the message to all that their participation and efforts are ongoing and continue to be acknowledged by the research team.

Recruitment of newborns took place over a period of 1 year for each pediatrician. This time span permitted us to avoid introducing bias related to the period of recruitment, for example, by recruiting newborns born during one season as opposed to another. The sample of children aged over 7 months reflects the distribution of births in Italy in terms of both geographic area (north, center, and south) and 21 clusters, based on the ISTAT data. Collection of data at the national level will 
permit the identification of differences in health care quality, such as those caused by socioeconomic inequalities present between the north and south of Italy [30], and of differences in family behaviors that influence child health status (eg, smoking or reading out loud to children). Better identifying health care-related inequalities will permit the channeling of resources where they are most needed [31]. If funding is obtained, the population enrolled could be expanded further.

As explained previously, the web portal has multiple functions and is fundamental for several reasons. The innovative aspects involve permitting the accurate input and monitoring of data through the use of a tool that creates data collection based on an $\mathrm{xml}$ definition, and providing pediatricians with interactive charts of current data to share with the children's parents.

This xml-based system allows a continuous and simple updating of the CRF, saving a lot of time in the development and testing phase. In addition, saving the data in the JavaScript Object Notation (JSON) format allows greater flexibility in the database structure which, therefore, does not need to be remodified at each CRF update [32].

Furthermore, our idea for the future is to interconnect the portal with apps for parents to use to access data and to provide additional information.

\section{Strengths and Limitations}

This is one of a very limited number of child cohorts based on the participation of family pediatricians, permitting the collection of data by those directly involved with the children and the implementation of findings to inform and help those directly involved (the children and their families). Furthermore, the large, representative population sample of newborns throughout the country, which allows stratified trends based on socioeconomic and geographic characteristics to be performed, and the use of standard measurements for anthropometric and neurocognitive parameters are among the strengths of this study.

A limit of the NASCITA cohort is that it does not collect biological samples due to the costs of data collection and storage, so it will not be able to evaluate genetic or immunological factors, for example. Resources and efforts were utilized, however, to achieve the largest population size possible in order to have enough power to study relatively common child exposures and outcomes. Another limitation is the potential bias in the pediatrician population because participation was voluntary and this may have led more motivated pediatricians to participate than others.

\section{Conclusions}

The NASCITA cohort is well underway and its population size will permit significant conclusions to be drawn. The key role of pediatricians in obtaining clinical data directly, along with the national-level representativity, will make the findings even more solid. In addition to promoting accurate data, the multiple functions of the web portal, with its expanding, interactive platform, will help maintain a solid relationship with the pediatricians and keep parents informed and interested in participating.

\section{Acknowledgments}

The authors thank all the participating pediatricians and committee members for their work and dedication. We also thank Davide Bazzi and Massimo Vitali for assistance in creating and in the support of servers, databases, and back-up system. No direct funding was received. The costs were covered by resources from the Laboratory for Mother and Child Health and by an economic contribution by the Associazione Amici del Mario Negri and by 2 anonymous donors, all of whom we wish to thank.

\section{Authors' Contributions}

MB, AC, and C Pansieri conceptualized the study, designed the web portal, and designed the CRF. AC, C Pandolfini, C Pansieri, and DM tested the CRF. MZ created the web portal, the eCRFs, and the interactive charts system. AC, MC, and C Pansieri coordinated and monitored data collection. MGC collaborated in assisting the pediatricians with data input problems and managed email and phone communication with pediatricians and with the technical and scientific committees. MC carried out the analyses. $\mathrm{DM}$ and $\mathrm{C}$ Pansieri were responsible for merging requests for modifications and corrections to the CRFs during testing, for re-testing them, and for creating the layout with data check and limit specifications for transformation into the electronic version. DM was responsible for website content and created the information cards for parents and the newsletters for the pediatricians. $\mathrm{C}$ Pandolfini wrote the first draft of the manuscript. All authors interpreted data and critically reviewed and revised the final manuscript as submitted. All authors agree to be accountable for aspects of their contribution to the work. MB is the guarantor.

\section{Conflicts of Interest}

None declared.

\section{References}

1. Black MM, Walker SP, Fernald LCH, Andersen CT, DiGirolamo AM, Lu C, Lancet Early Childhood Development Series Steering Committee. Early childhood development coming of age: science through the life course. Lancet 2017 Jan 07;389(10064):77-90 [FREE Full text] [doi: 10.1016/S0140-6736(16)31389-7] [Medline: 27717614]

2. Barouki R, Gluckman PD, Grandjean P, Hanson M, Heindel JJ. Developmental origins of non-communicable disease: implications for research and public health. Environ Health 2012 Jun 27;11:42 [FREE Full text] [doi: 10.1186/1476-069X-11-42] [Medline: 22715989] 
3. Fleming TP, Watkins AJ, Velazquez MA, Mathers JC, Prentice AM, Stephenson J, et al. Origins of lifetime health around the time of conception: causes and consequences. Lancet 2018 May 05;391(10132):1842-1852 [FREE Full text] [doi: 10.1016/S0140-6736(18)30312-X] [Medline: 29673874]

4. Larsen PS, Kamper-Jørgensen M, Adamson A, Barros H, Bonde JP, Brescianini S, et al. Pregnancy and birth cohort resources in europe: a large opportunity for aetiological child health research. Paediatr Perinat Epidemiol 2013 Jul;27(4):393-414. [doi: 10.1111/ppe.12060] [Medline: 23772942]

5. Thompson L, Kemp J, Wilson P, Pritchett R, Minnis H, Toms-Whittle L, et al. What have birth cohort studies asked about genetic, pre- and perinatal exposures and child and adolescent onset mental health outcomes? A systematic review. Eur Child Adolesc Psychiatry 2010 Jan;19(1):1-15 [FREE Full text] [doi: 10.1007/s00787-009-0045-4] [Medline: 19636604]

6. Baxter-Jones AD, Cardy AH, Helms PJ, Phillips DO, Smith WC. Influence of socioeconomic conditions on growth in infancy: the 1921 Aberdeen birth cohort. Arch Dis Child 1999 Jul;81(1):5-9 [FREE Full text] [doi: 10.1136/adc.81.1.5] [Medline: 10373124$]$

7. Framingham Heart Study. URL: https://framinghamheartstudy.org/ [accessed 2021-02-16]

8. Nurses' Health Study. URL: https://www.nurseshealthstudy.org/ [accessed 2021-02-16]

9. Pearson H. Children of the 90s: Coming of age. Nature 2012 Apr 11;484(7393):155-158. [doi: 10.1038/484155a] [Medline: 22498607]

10. Birthcohorts.net. URL: https://www.birthcohorts.net/ [accessed 2021-02-16]

11. Porta D, Fantini M. Prospective cohort studies of newborns in Italy to evaluate the role of environmental and genetic characteristics on common childhood disorders. Ital J Pediatr 2006 Dec;32:350-357.

12. Porta D, Forastiere F, Di Lallo D, Perucci CA, Grupo Collaborativo GASPII. [Enrolment and follow-up of a birth cohort in Rome]. Epidemiol Prev 2007;31(6):303-308 [FREE Full text] [Medline: 18326421]

13. Lanari M, Vandini S, Adorni F, Prinelli F, Di Santo S, Silvestri M, Study Group of Italian Society of Neonatology on Risk Factors for RSV Hospitalization. Prenatal tobacco smoke exposure increases hospitalizations for bronchiolitis in infants. Respir Res 2015 Dec 22;16:152 [FREE Full text] [doi: 10.1186/s12931-015-0312-5] [Medline: 26695759]

14. Barchitta M, Maugeri A, La Rosa MC, Magnano San Lio R, Favara G, Panella M, et al. Single Nucleotide Polymorphisms in Vitamin D Receptor Gene Affect Birth Weight and the Risk of Preterm Birth: Results From the. Nutrients 2018 Aug 27;10(9):1172 [FREE Full text] [doi: 10.3390/nu10091172] [Medline: 30150529]

15. Brescianini S, Fagnani C, Toccaceli V, Medda E, Nisticò L, D'Ippolito C, et al. An update on the Italian Twin Register: advances in cohort recruitment, project building and network development. Twin Res Hum Genet 2013 Feb;16(1):190-196. [doi: 10.1017/thg.2012.85] [Medline: 23088847]

16. Richiardi L, Baussano I, Vizzini L, Douwes J, Pearce N, Merletti F. Feasibility of recruiting a birth cohort through the Internet: the experience of the NINFEA cohort. Eur J Epidemiol 2007;22(12):831-837. [doi: 10.1007/s10654-007-9194-2] [Medline: 17955333]

17. Farchi S, Forastiere F, Vecchi Brumatti L, Alviti S, Arnofi A, Bernardini T, et al. Piccolipiù, a multicenter birth cohort in Italy: protocol of the study. BMC Pediatr 2014 Feb 07;14:36 [FREE Full text] [doi: 10.1186/1471-2431-14-36] [Medline: 24506846]

18. Valent F, Mariuz M, Bin M, Little D, Mazej D, Tognin V, et al. Associations of prenatal mercury exposure from maternal fish consumption and polyunsaturated fatty acids with child neurodevelopment: a prospective cohort study in Italy. J Epidemiol 2013 Sep 05;23(5):360-370 [FREE Full text] [doi: 10.2188/jea.je20120168] [Medline: 23933621]

19. Lanari M, Prinelli F, Adorni F, Di Santo S, Vandini S, Silvestri M, Study Group of Italian Society of Neonatology on Risk Factors for RSV Hospitalization. Risk factors for bronchiolitis hospitalization during the first year of life in a multicenter Italian birth cohort. Ital J Pediatr 2015 May 26;41:40 [FREE Full text] [doi: 10.1186/s13052-015-0149-z] [Medline: 26006025]

20. Bonati M, Campi R. What can we do to improve child health in southern Italy? PLoS Med 2005 Sep;2(9):e250 [FREE Full text] [doi: 10.1371/journal.pmed.0020250] [Medline: 16104833]

21. Landi S, Ivaldi E, Testi A. Socioeconomic status and waiting times for health services: An international literature review and evidence from the Italian National Health System. Health Policy 2018 Apr;122(4):334-351. [doi: 10.1016/j.healthpol.2018.01.003] [Medline: 29373188]

22. Pansieri C, Clavenna A, Pandolfini C, Zanetti M, Calati MG, Miglio D, et al. NASCITA Italian birth cohort study: a study protocol. BMC Pediatr 2020 Feb 19;20(1):80 [FREE Full text] [doi: 10.1186/s12887-020-1961-1] [Medline: $\underline{32075601]}$

23. Nascere e crescere in Italia (Coorte NASCITA). URL: http://nascita.marionegri.it/ [accessed 2021-02-16]

24. Istituto Italiano di Statistica (ISTAT). Main geographic statistics on cities. URL: https//www.istat.it/it/archivio/156224 [accessed 2021-02-16]

25. Corsello G, Ferrara P, Chiamenti G, Nigri L, Campanozzi A, Pettoello-Mantovani M. The Child Health Care System in Italy. J Pediatr 2016 Oct;177S:S116-S126. [doi: 10.1016/j.jpeds.2016.04.048] [Medline: 27666260]

26. Bonati M, Reale L, Zanetti M, Cartabia M, Fortinguerra F, Capovilla G, Lombardy ADHD Group. A Regional ADHD Center-Based Network Project for the Diagnosis and Treatment of Children and Adolescents With ADHD. J Atten Disord 2018 Oct;22(12):1173-1184. [doi: 10.1177/1087054715599573] [Medline: 26320120] 
27. Zanetti M, Campi R, Olivieri P, Campiotti M, Faggianelli A, Bonati M. A Web-Based Form With Interactive Charts Used to Collect and Analyze Data on Home Births in Italy. J Med Internet Res 2019 Mar 22;21(3):e10335 [FREE Full text] [doi: 10.2196/10335] [Medline: $\underline{30900993]}$

28. GitLab. Gatherings Crafter. URL: https://gitlab.com/dellaltromondo/gatherings-crafter [accessed 2020-07-20]

29. Pansieri C, Pandolfini C, Clavenna A, Choonara I, Bonati M. An Inventory of European Birth Cohorts. Int J Environ Res Public Health 2020 Apr 28;17(9):3071 [FREE Full text] [doi: 10.3390/ijerph17093071] [Medline: 32354101]

30. de Waure C, Bruno S, Furia G, Di Sciullo L, Carovillano S, Specchia ML, et al. Health inequalities: an analysis of hospitalizations with respect to migrant status, gender and geographical area. BMC Int Health Hum Rights 2015 Feb 07;15:2 [FREE Full text] [doi: 10.1186/s12914-014-0032-9] [Medline: 25881154]

31. Lauria L, Spinelli A, Buoncristiano M, Nardone P. Decline of childhood overweight and obesity in Italy from 2008 to 2016 : results from 5 rounds of the population-based surveillance system. BMC Public Health 2019 May 21;19(1):618 [FREE Full text] [doi: 10.1186/s12889-019-6946-3] [Medline: 31113403 ]

32. JavaScript Object Notation. URL: https://www.w3.org/TR/html-json-forms/\#the-application-json-encoding-algorithm [accessed 2021-02-16]

\author{
Abbreviations \\ ACP: Pediatric Cultural Association \\ CRF: case report form \\ eCRF: electronic case report form \\ ISTAT: National Statistics Institute \\ JSON: JavaScript Object Notation \\ NASCITA cohort: NAscere e creSCere in ITAlia cohort
}

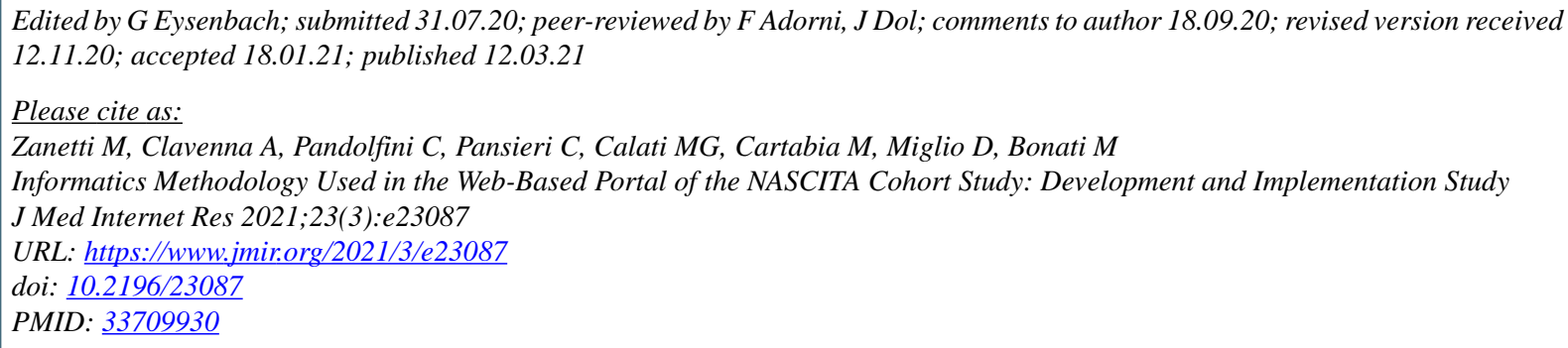

(CMichele Zanetti, Antonio Clavenna, Chiara Pandolfini, Claudia Pansieri, Maria Grazia Calati, Massimo Cartabia, Daniela Miglio, Maurizio Bonati. Originally published in the Journal of Medical Internet Research (http://www.jmir.org), 12.03.2021. This is an open-access article distributed under the terms of the Creative Commons Attribution License (https://creativecommons.org/licenses/by/4.0/), which permits unrestricted use, distribution, and reproduction in any medium, provided the original work, first published in the Journal of Medical Internet Research, is properly cited. The complete bibliographic information, a link to the original publication on http://www.jmir.org/, as well as this copyright and license information must be included. 